\title{
Discovery of Ground-state Absorption Line Polarization and Sub-Gauss Magnetic Field in the Post-AGB Binary System 89 Her
}

\author{
Heshou Zhang ${ }^{1,2}$ (iD), Manuele Gangi ${ }^{3,4}$ (D) , Francesco Leone ${ }^{5}$ (D) Andrew Taylor $^{1}$, and Huirong Yan $^{1,2,6}$ (D) \\ ${ }^{1}$ Deutsches Elektronen-Synchrotron DESY, Platanenallee 6, D-15738 Zeuthen, Germany; huirong.yan@desy.de \\ ${ }^{2}$ Institut für Physik und Astronomie, Universität Potsdam, Haus 28, Karl-Liebknecht-Str. 24/25, D-14476 Potsdam, Germany \\ INAF - Osservatorio Astrofisico di Catania, Via S. Sofia 78, I-95123 Catania, Italy \\ ${ }^{4}$ INAF - Osservatorio Astronomico di Roma, Via Frascati 33, I-00078 Monte Porzio Catone, Italy \\ ${ }^{5}$ Universitá di Catania, Dipartimento di Fisica e Astronomia, Sezione Astrofisica, Via S. Sofia 78, I-95123 Catania, Italy \\ Received 2020 February 4; revised 2020 July 20; accepted 2020 September 14; published 2020 October 7
}

\begin{abstract}
We report the polarization of ground-state absorption lines from the post-asymptotic giant branch (post-AGB) binary star system 89 Herculis $(89$ Her). Two ground-state neutral iron lines are found to have counterintuitive high-amplitude polarizations and an unchanged polarization direction through the orbital period. This is contrary to the pattern of polarization of absorption lines from excited states, which are synchronized with the orbital phase due to optical pumping. This can be explained by magnetic realignment of the ground state, whereby the 3D mean magnetic field is unveiled from the degree and direction of the polarizations of the two iron lines. The field strength is also constrained to be $\lesssim 100 \mathrm{mG}$. Our result improves the accuracy by orders of magnitude compared to the previous $10 \mathrm{G}$ upper limit set by non-detection of the Zeeman effect.
\end{abstract}

Unified Astronomy Thesaurus concepts: Starlight polarization (1571); Spectropolarimetry (1973); Stellar photospheres (1237); Stellar properties (1624); Stellar spectral lines (1630); Astrophysical magnetism (102); Stellar magnetic fields (1610)

\section{Introduction}

Spectral polarimetry observations can provide extensive information on magnetic field and the topology of radiation structure. Developments in high-resolution spectral facilities with polarimeters (e.g., the Potsdam Echelle Polarimetric and Spectroscopic Instrument (PEPSI), Strassmeier et al. 2008; the High Accuracy Radial velocity Planet Searcher (HARPS), Piskunov et al. 2011; the HARPS-North polarimeter (HANPO), Leone et al. 2014) facilitate the investigation of polarimetric properties of single spectral lines. The surprisingly high polarimetric signals shown by the prototypical star 89 Herculis (89 Her; on average, more than 1\%) give us a unique opportunity to study this system (Leone et al. 2018).

$89 \mathrm{Her}$ is a post-asymptotic giant branch (post-AGB) binary system. The primary star is an F-type supergiant with a radius $R_{\text {pri }}=41 R_{\odot}$ and an effective temperature $T_{\text {eff }}=6500 \mathrm{~K}$, while the secondary is an M-type main sequence with $R_{\mathrm{sec}}=0.6 R_{\odot}$ and $T_{\text {eff }}=4045 \mathrm{~K}$. The radius of the secondary orbital track is $r_{\text {orb }}=67 R_{\odot} .89$ Her has an orbital period $P_{\text {orb }} \sim 288$ days and inclination $12^{\circ}$. It exhibits a circumstellar environment consisting of two main components: an expanding hourglass structure and a circumbinary rotating disk (Waters et al. 1993; Bujarrabal et al. 2007; Kipper 2011). The projection of stellar outflows on the picture plane is $45^{\circ}$ compared to the east-west orientation (Bujarrabal et al. 2007). Leone et al. (2018) found that linearly polarized photospheric absorption lines of 89 Her present $Q / I$ and $U / I$ signals that vary according to the secondary orbital period (hereafter "orbital synchronization"). They have excluded the origin of such a signal from either the depolarization of stellar continuum polarization (including pulsations and hot spots) or scattered polarization from a bipolar outflows, and found that it is a result of optical pumping in the stellar environment.

\footnotetext{
${ }^{6}$ Corresponding Author.
}

In this Letter, we report the discovery of two ground-state Fe I photosphere absorption line polarizations whose directions have not shown orbital synchronization, but rather are aligned through all orbital phases. ${ }^{7}$ This observation indicates the existence of groundstate magnetic alignment, which was proposed theoretically as a magnetic tracer in Yan \& Lazarian (2006). The alignment is in terms of the angular momentum of atoms and ions (hereafter "atoms" for simplicity). Ground-state alignment (GSA) is an established physical phenomenon that has solid physical foundations and has been studied and supported by numerous experiments (Kastler 1950; Brossel et al. 1952; Hawkins \& Dicke 1953; Hawkins 1955; Cohen-Tannoudji et al. 1969). The anisotropic radiation aligns the atoms in the ground state by optical pumping (Happer 1972; Varshalovich 1971; Landolfi \& Landi Degl'Innocenti 1986). These radiative-aligned atoms are magnetically realigned by fast precession as long as the Larmor precession rate $\nu_{\mathrm{Lar}}$ is larger than the radiative pumping rate $\nu_{\mathrm{Rad}}$ (Yan \& Lazarian 2006, 2007). In the GSA regime, the atoms are aligned with radiation field or realigned by magnetic field depending on the ratio of the two rates $\left(r_{\mathrm{A}} \equiv \nu_{\mathrm{Lar}} / \nu_{\mathrm{Rad}}\right)$. In the Ground-level Hanle regime, however, the atoms preferentially follow neither the magnetic or radiation field ( $r_{\mathrm{A}} \sim 1$; Yan \& Lazarian 2008). The GSA probe is particularly suitable for the sub-Gauss magnetic field because the atoms have long lifetimes in their ground states. The resulting absorption lines from the aligned atoms are polarized parallel or perpendicular to the magnetic field direction.

The magnetic field in the 89 Her's primary has been unknown to date. Earlier attempts with current magnetic tracers have not provided strong constraint; no continuum polarization has been detected (see Akras et al. 2017), and previous spectropolarimetric studies have revealed that the source is circularly unpolarized, which only implies an upper limit of $10 \mathrm{G}$ (Sabin et al. 2015). In this Letter, first we will discuss the observational results and then

\footnotetext{
7 Hereafter, we will assume the same ephemeris adopted in Leone et al. (2018) to compute the orbital phases of 89 Her (iph).
} 

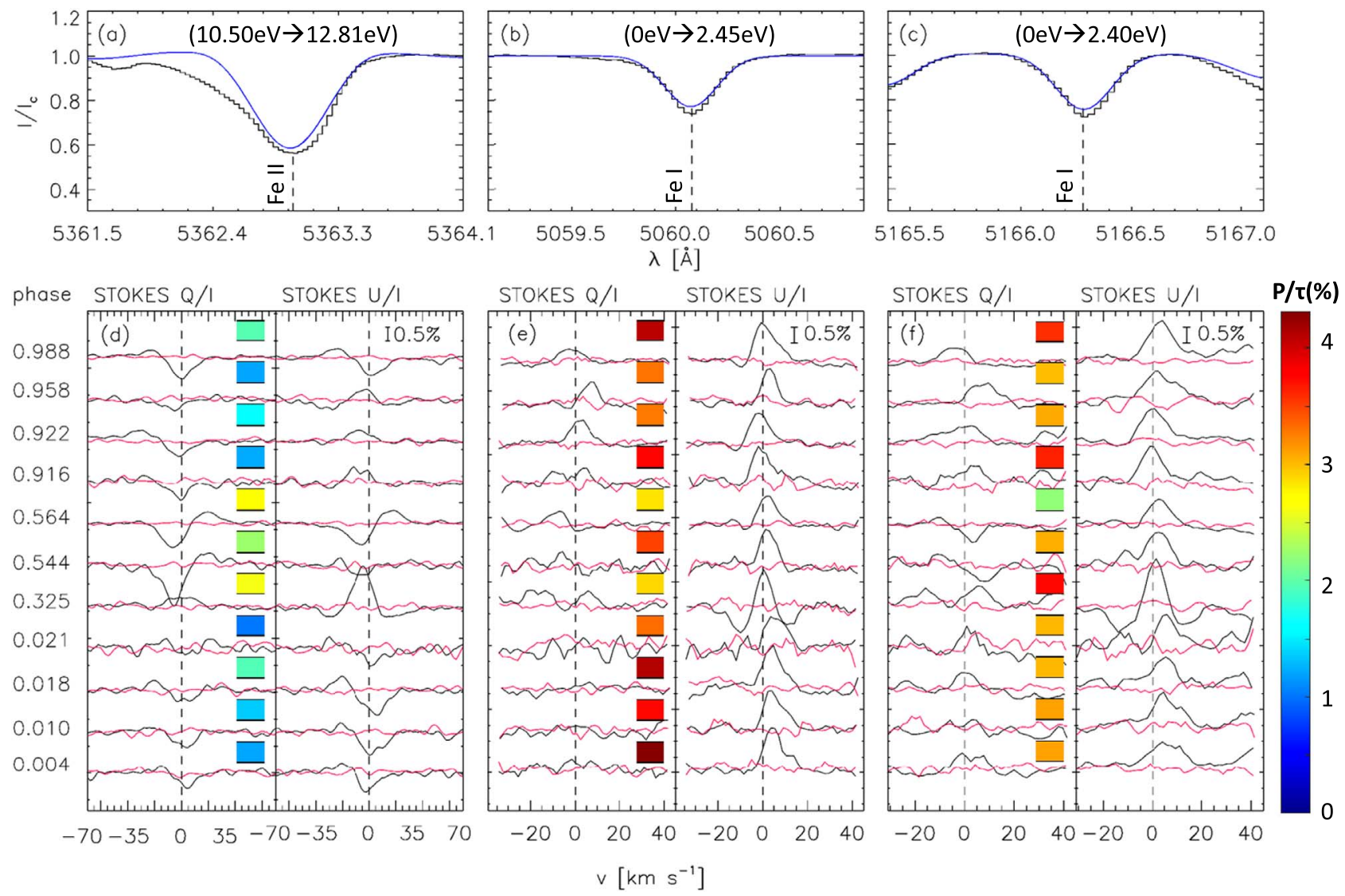

Figure 1. Top row: (a) the observed spectral line profile (black) and its comparison with the photospheric lines modeled (blue) using the synthetic spectrum package SYNTHE (Kurucz 2005) for the radiative dominant line Fe II $\lambda 5362.970 \AA$ (a), and of the two magnetic aligned lines Fe I $\lambda 5060.249 \AA$ (b) and Fe I $\lambda 5166.282 \AA$ (c). These lines are unblended and identified from the photosphere. Bottom row: $Q / I$ and $U / I$ profiles of (d) Fe II $\lambda 5362.970 \AA$, (e) $\mathrm{Fe}$ I $\lambda 5060.249 \AA$, (f) Fe I $\lambda 5166.282$ $\AA$ lines. The null spectra are marked in red. The degree of polarization $\mathrm{P}$ in each phase is denoted by the color mark.

Table 1

3D Magnetic Field Angles

\begin{tabular}{lcccc}
\hline \hline iph & $\xi_{B}$ & $\Delta \xi_{B}$ & $\theta_{B}$ & $\Delta \theta_{B}$ \\
\hline 0.004 & 35.5 & 7.9 & 62.4 & 11.0 \\
0.01 & 41.5 & 10.0 & 56.8 & 15.9 \\
0.018 & 36.8 & 6.6 & 58.6 & 13.6 \\
0.021 & 40.5 & 16.6 & 54.9 & 14.6 \\
0.325 & 38.7 & 8.5 & 55.6 & 11.9 \\
0.544 & 50.2 & 9.7 & 59.3 & 11.1 \\
0.564 & 54.0 & 8.0 & 53.2 & 11.0 \\
0.916 & 35.0 & 12.3 & 57.0 & 15.6 \\
0.922 & 30.9 & 7.2 & 54.5 & 13.8 \\
0.958 & 34.1 & 8.1 & 56.0 & 14.3 \\
0.988 & 36.7 & 4.7 & 67.6 & 8.3 \\
\hline
\end{tabular}

Note. The POS component $\xi_{B}$ and LOS component $\theta_{B}$ as demonstrated in Figure 2(c). $\Delta \xi_{B}$ is the $3 \sigma$ error bar obtained from the observed direction of polarization, and $\Delta \theta_{B}$ is the $1 \sigma$ error bar inferred from the degree of the polarization (Section 4.1)

provide theoretical interpretations, which lead to the extraction of the mean magnetic field information on the photosphere of 89 Her's primary.

\section{Observational Data}

Reduced spectropolarimetric data of 89 Her have been collected at the 3.6-m Canada-France-Hawaii Telescope with the Echelle SpectroPolarimetric Device for the Observation of Stars
(ESPaDOnS: $\mathrm{R}=68000$; Donati et al. 2006). They consist of Stokes $I, Q, U$, and null $N Q, N U$ (Leone et al. 2016). From these data we computed the polarization $P$, the null polarization $N P$, and the polarization angle $\xi$, according to the definitions given in Landi Degl'Innocenti \& Landolfi (2004). The spectral lines studied are unblended with other lines, as shown in Figures 1(a)-(c). ${ }^{8}$ To provide an objective criterion for deciding whether a polarimetric signal is detected across the spectral lines, we follow the statistical test of Donati et al. (1997). First we computed the reduced $\chi^{2}$ inside and outside the spectral lines for both $P$ and null NP profiles. Then we calculated the $\chi^{2}$ detection probabilities based on the achieved signal-to-noise ratio $(\mathrm{S} / \mathrm{N})$, as shown in Table 1 . We concentrate on the central part of the absorption line (corresponding to the marked green zone in Figure 2(d)), which is least influenced by the blue and redshifted emission from outflows.

\section{Polarization Analysis for the Photospheric Absorption Lines}

Previous spectropolarimetric works focus only on lines with a relatively large optical depth $(\tau \gtrsim 0.5)$. We found that all of the photosphere lines showing orbital synchronization" stand for the transitions between the excited states. As an example, the linear polarization profile of Fe II $\lambda 5362.970 \AA(10.50 \mathrm{eV} \rightarrow 12.81 \mathrm{eV})$ is presented in Figure 1(d), showing the correlation between their

\footnotetext{
8 Unlike $\mathrm{Na} \mathrm{D}$ lines and Balmer lines in $89 \mathrm{Her}$, where the photospheric line is blended with multiple blueshifted absorption components as well as redshifted strong interstellar absorption (see, e.g., Kipper 2011; Gangi \& Leone 2019).
} 

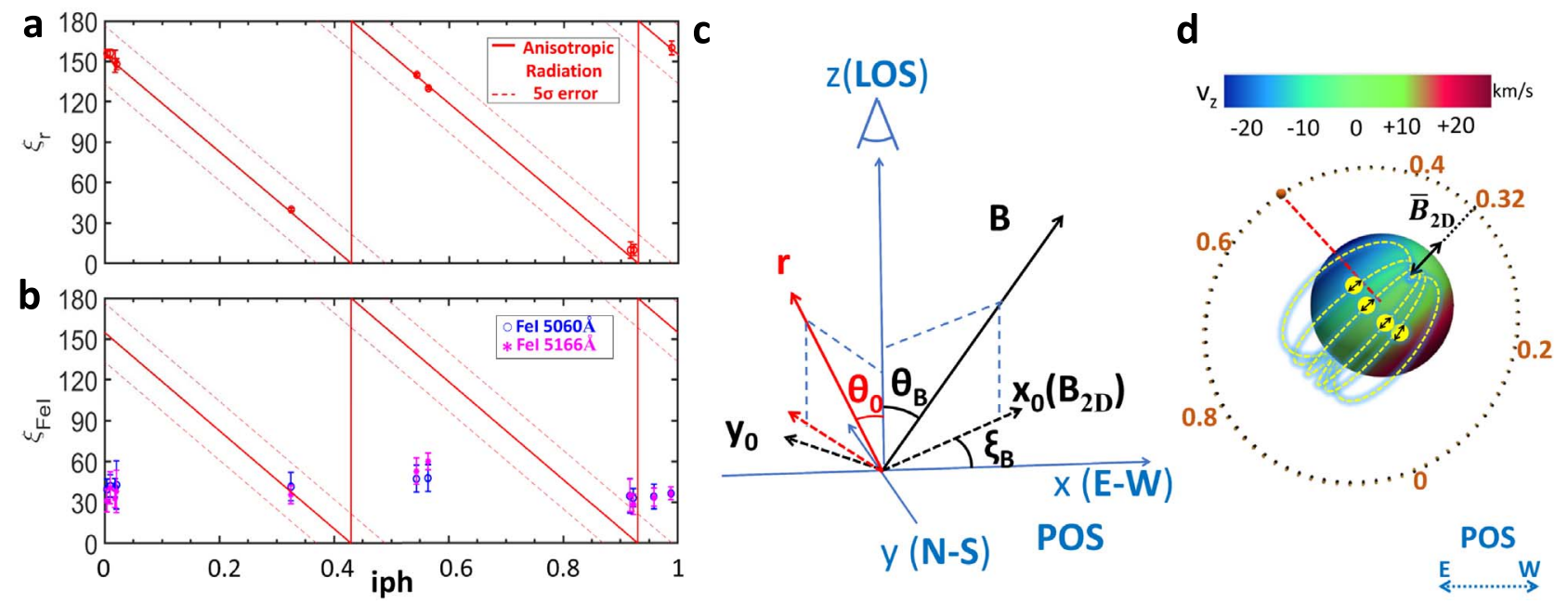

Figure 2. (a) Strong absorption lines between excited states; the polarization angle $\left(\xi_{r}\right)$ shows orbital synchronization. iph is orbital phase. (b) The polarization angle $\xi_{\mathrm{FeI}}$ for the two Fe I lines. The error bars mark the $3 \sigma$ uncertainty range. (c) 3D view of the system showing the reference frame. The $x y z$-frame is the observational frame where the oxaxis is defined arbitrarily on east-west direction. $\theta_{B}$ and $\xi_{B}$ are the polar and azimuth angles for magnetic field in the frame. The $x_{0} y_{0} z$-frame is the theoretical frame where the $\mathrm{ox}_{0}$-axis is the direction of the plane-of-sky (POS) magnetic field projection. (d) 89 Her system. The orbital phases are marked along the secondary track. The color scale denotes the line-of-sight (LOS) velocity $\left(v_{z}\right)$ of the photosphere medium. Our analysis measures the mean magnetic field in the green region of the primary's photosphere, corresponding to the central part of the absorption lines. A possible magnetic tomography of the system is presented here. The yellow dashed lines are magnetic field lines. The double arrows are the polarization directions. The average magnetic field projection on POS $\bar{B}_{2 D}$ is $\sim 45^{\circ}$ to the east-west direction pointing to the orbital phase 0.32 .
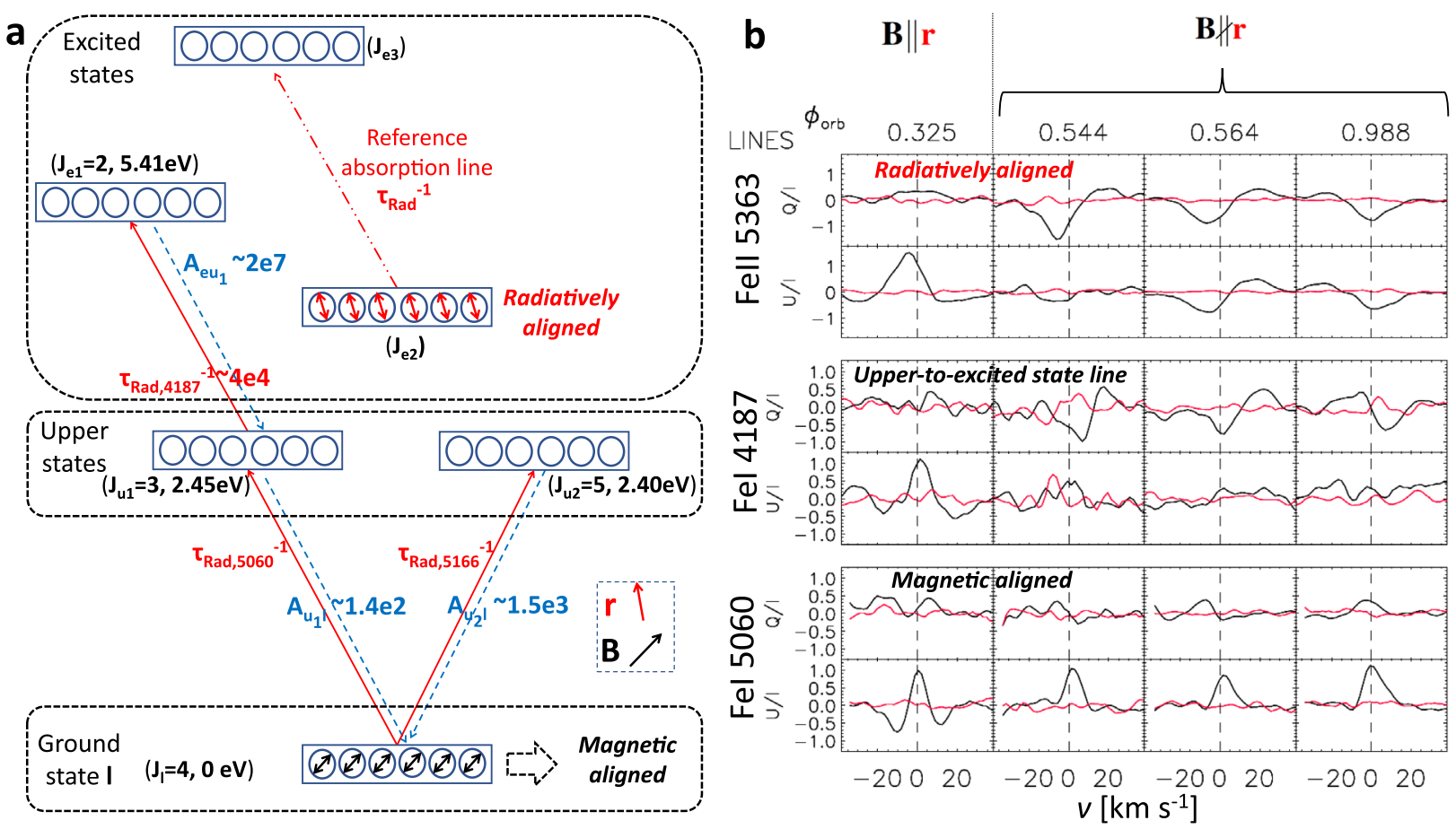

Figure 3. (a) Schematics of the optical pumping and magnetic realignment in the GSA regime. Rectangles with circles represent the energy state occupied by atoms. The arrows are the atomic angular momentum. The ground state is magnetically aligned. "The upper states" mark the upper levels for transitions of focus Fe I $\lambda \lambda 5060$, $5166 \AA$. Other states are marked as excited states. The Einstein coefficients for transitions involving the level Fe I $2.45 \mathrm{eV}$ are marked. (b) Stokes parameters of the selected absorption lines. They are the pumping reference Fe II $\lambda 5362.970 \AA$, Fe I $\lambda 4187.039 \AA$ from "the upper state" $2.45 \mathrm{eV}$, and the GSA line Fe I $\lambda 5060.249 \AA$ at four different orbital phases: $0.325,0.544,0.564$, and 0.988 .

polarization angle and the vector connecting the two stellar bodies during their orbits (see Figure 2(a)).

Here we focus on two ground-state neutral iron absorption lines, Fe I $\lambda 5060.249 \AA(0 \mathrm{eV} \rightarrow 2.45 \mathrm{eV})$ and Fe I $\lambda 5166.282 \AA$ $(0 \mathrm{eV} \rightarrow 2.40 \mathrm{eV})$. These lines are weak $(\tau \sim 0.2$; see Figures 1(b), (c)) and therefore were previously ignored. As demonstrated in Figures 1(e), (f), they have shown counterintuitive strong polarization signatures. Moreover, the polarization angles of these two Fe I lines are aligned through all orbital phases (see Figure 2(b)) at $\sim 45^{\circ}$ toward an east-west orientation. Such results indicate that the atomic angular momentum on the ground state is realigned by fast magnetic precession; in other words, the magnetic realignment dominates over optical pumping. We note that at $i p h=0.325$, the polarization direction of the radiation dominant lines are the same as that of GSA lines. This is because the radiative pumping direction at this phase coincides with the magnetic alignment direction. 
We find that it is important to account only for the absorption term of linear polarization in the analysis of these two GSA lines. The reasons are as follows. (1) The background stellar continuum is unpolarized (Akras et al. 2017). (2) The spontaneous (scattered) emission from "the upper states" are negligible (see Figure 3(a) for the microphysics). The upper states of those two transitions have different angular momentum $\left(J_{u}=3,5\right.$, respectively). Hence the scattered emission would yield a different polarization profile between the two lines at different iph. This is at odds with the observations of the aligned polarization direction. (3) The polarization signals for absorption from "the upper states" also support our conclusion (see Figure 3(b)). We consider the absorption line Fe I $\lambda 4187.039 \AA(2.45 \mathrm{eV} \rightarrow 5.41 \mathrm{eV})$, i.e., the absorption from "the upper level" $2.45 \mathrm{eV}$. Its Stokes $Q$ and $U$ signals are compared with two types of lines: "the pumping-dominant reference" Fe II $\lambda 5362.970 \AA$ and the GSA line Fe I $\lambda 5060.249 \AA$. The orbital variation pattern of Fe I $\lambda 4187.039 \AA(2.45 \mathrm{eV} \rightarrow 5.41 \mathrm{eV})$ is similar to the Fe II $\lambda 5362.970 \AA$ line, showing a varying radiative alignment of atoms on $2.45 \mathrm{eV}$. Therefore, this level's varying contribution to the unchanged polarization signal of the ground-state absorption line Fe II $\lambda 5060.249 \AA$ is negligible. Magnetic alignment happens only in the ground state.

\section{Theoretical Analyses}

\subsection{Theoretical Expectations and the Identification of the $2 D$ Magnetic Field}

We solve the atomic transition equations to estimate the expected level of polarization signals from 89 Her (see Appendix B for details). The ground state is taken to be fully magnetically aligned and the anisotropic pumping is provided by the secondary. The $90^{\circ}$-ambiguity (known as the Van Vleck ambiguity; Van Vleck 1925; House 1974) between the observed polarization and the position of sky (POS) magnetic projection is also resolved from the comparison of theoretical expectations and the observed degree of polarization. We scan the full parameter space to solve the expected maximum alignment parameter and the ratio of polarization $P / \tau$. Given the face-on orbit, we take the radiation from the secondary in the range $\theta_{0} \in\left[65^{\circ}, 90^{\circ}\right]$. We find that in the case of parallel alignment $\left(\varepsilon_{\sigma}=+1\right)$, the expected maximum polarization signal can reach $P_{\max } / \tau(5060 \AA) \gtrsim 8 \%$ and $P_{\max } / \tau(5166 \AA) \gtrsim 4 \%$, respectively. These upper limits for the two lines are consistent with the observed polarization signals of both ground-state absorption lines. Nonetheless, the maximum $P_{\max } / \tau$ in the case of perpendicular alignment $\left(\varepsilon_{\sigma}=-1\right)$ calculated from the transitional equation are $4.9 \%$ and $2.5 \%$ for the absorption lines $\lambda 5060.249 \AA$ and $\lambda 5166.249 \AA$, respectively, smaller than the observed values. The perpendicular alignment is thus excluded.

\subsection{Magnetic Field in the Third Dimension}

The theoretical Stokes parameters $[\tilde{I}, \tilde{Q}, \tilde{U}, \tilde{V}]$ are defined with $\tilde{Q}$ measured from the magnetic field direction on the POS (see $x_{0} y_{0} z$-frame Figure 2(c)). In the case of purely absorbing medium, they are given by Yan \& Lazarian (2006):

$$
\begin{aligned}
\tilde{I} & =\left(I_{0}+Q_{0}\right) e^{-\tau\left(1+\eta_{1} / \eta_{0}\right)}+\left(I_{0}-Q_{0}\right) e^{-\tau\left(1-\eta_{1} / \eta_{0}\right)}, \\
\tilde{Q} & =\left(I_{0}+Q_{0}\right) e^{-\tau\left(1+\eta_{1} / \eta_{0}\right)}-\left(I_{0}-Q_{0}\right) e^{-\tau\left(1-\eta_{1} / \eta_{0}\right)}, \\
\tilde{U} & =U_{0} e^{-\tau}, \tilde{V}=V_{0} e^{-\tau},
\end{aligned}
$$

where $\eta_{i}$ are absorption coefficients of the Stokes parameters. Theoretically, the absorption coefficients are determined by (see Landi Degl'Innocenti 1984):

$$
\begin{aligned}
\eta_{i}(\nu, \Omega)= & \frac{h \nu_{0}}{4 \pi} B n\left(J_{l}\right) \xi\left(\nu-\nu_{0}\right) \sum_{k q}(-1)^{k} \\
& \times w_{J_{l} J_{u}}^{(k)} \sigma_{q}^{k}\left(J_{l}\right) \mathcal{J}_{q}^{k}(i, \Omega) .
\end{aligned}
$$

where $\sigma_{q}^{k}\left(J_{l}\right) \equiv \rho_{q}^{k}\left(J_{l}\right) / \rho_{0}^{0}\left(J_{l}\right), \rho_{q}^{k}\left(J_{l}\right)$ is the irreducible density matrix on the ground state. In the GSA regime, only $q=0$ is non-zero and $\sigma_{0}^{2}\left(J_{l}\right)$ is the alignment parameter (Yan \& Lazarian 2006). The quantity $n\left(J_{l}\right)$ is the population on the lower level. The quantity $\mathcal{J}_{q}^{k}(i, \Omega)$ is the irreducible unit tensor for Stokes parameters $I, Q$, and $U$. The ratio $\eta_{1} / \eta_{0}$ in the GSA regime is then (Yan \& Lazarian 2006):

$$
\eta_{1} / \eta_{0}=-\frac{1.5 \sigma_{0}^{2}\left(J_{l}\right) \sin ^{2} \theta_{B} w_{J_{l} J_{u}}^{(2)}}{\sqrt{2}+\sigma_{0}^{2}\left(J_{l}\right)\left(1-1.5 \sin ^{2} \theta_{B}\right) w_{J_{l} J_{u}}^{(2)}},
$$

where $\theta_{B}$ is the angle between magnetic field and the LOS.

$$
w_{J_{l} J_{u}}^{(2)} \equiv\left\{\begin{array}{lll}
1 & 1 & 2 \\
J_{l} & J_{l} & J_{u}
\end{array}\right\} /\left\{\begin{array}{ccc}
1 & 1 & 0 \\
J_{l} & J_{l} & J_{u}
\end{array}\right\},
$$

is determined by the electron configurations of the transition $J_{l} \rightarrow J_{u}$. The alignment parameter $\sigma_{0}^{2}$ is the same for two transitions with the same ground level $\left(J_{l} \rightarrow J_{u 1}, J_{l} \rightarrow J_{u 2}\right)$. Different observed degrees of polarization for these two lines result from the angular momentum configuration parameters $w_{J_{l} J_{u 1}}^{(2)}, w_{J_{l} J_{u 2}}^{(2)}$. Therefore, we obtained from Equation (3):

$$
\begin{aligned}
& \sigma_{0}^{2}\left(J_{l}\right)= \\
& \left\{\begin{array}{l}
\frac{\sqrt{2}\left(c_{1} c_{2}\left(w_{J_{l} J_{u 2}}^{(2)}-w_{J_{l} J_{u 1}}^{(2)}\right)+\varepsilon_{\sigma}\left(c_{1} w_{J_{l} J_{u 2}}^{(2)}-c_{2} w_{J_{l} J_{u 1}}^{(2)}\right)\right)}{\left(c_{2}-c_{1}\right) w_{J_{l} J_{u 1}}^{(2)} w_{J_{l} J_{u 2}}^{(2)}} \\
\operatorname{sgn}\left(w_{J_{l} J_{u 1}}^{(2)}\right)=\operatorname{sgn}\left(w_{J_{l} J_{u 2}}^{(2)}\right) ; \\
-\frac{\sqrt{2}\left(c_{1} c_{2}\left(w_{J_{l} l_{u 2}}^{(2)}-w_{J_{l} J_{u} 1}^{(2)}\right)+\varepsilon_{\sigma}\left(c_{1} w_{J_{l} J_{u 2}}^{(2)}+c_{2} w_{J_{l} J_{u 1}}^{(2)}\right)\right)}{\left(c_{1}+c_{2}\right) w_{J_{l} J_{u 1}}^{(2)} w_{J_{l} J_{u 2}}^{(2)}} \\
\operatorname{sgn}\left(w_{J_{l} J_{u 1}}^{(2)}\right)=-1, \operatorname{sgn}\left(w_{J_{l} J_{u 2}}^{(2)}\right)=+1 ; \\
\sigma_{0}^{2}\left(J_{l}\right) \sin ^{2} \theta_{B}= \\
\frac{2 \sqrt{2} c_{1} c_{2}\left(w_{J_{l} J_{u 2}}^{(2)}-w_{J_{l} J_{u 1}}^{(2)}\right)}{3\left(c_{2}-c_{1}\right) w_{J_{l} J_{u 1}}^{(2)} w_{J_{l} J_{u 2}}^{(2)}}, \\
\operatorname{sgn}\left(w_{J_{l} J_{u 1}}^{(2)}\right)=\operatorname{sgn}\left(w_{J_{l} J_{u 2}}^{(2)}\right) ; \\
-\frac{2 c_{1} c_{2}\left(w_{J_{l} J_{u 2}}^{(2)}-w_{J_{l}}^{(2)}\right)}{3\left(c_{1}+c_{2}\right) w_{J_{l} J_{u 1}}^{(2)} w_{J_{l} J_{u 2}}^{(2)},} \\
\operatorname{sgn}\left(w_{J_{l} J_{u 1}}^{(2)}\right)=-1, \operatorname{sgn}\left(w_{J_{l} J_{u 2}}^{(2)}\right)=+1 ;
\end{array}\right.
\end{aligned}
$$

The ratio $c_{j} \equiv\left|\eta_{1} / \eta_{0}\right|=\frac{1}{2 \tau_{j}} \ln \left(\frac{1+P_{j}}{1-P_{j}}\right), j=1,2$ is directly related to the degrees of polarization and optical depths of a 


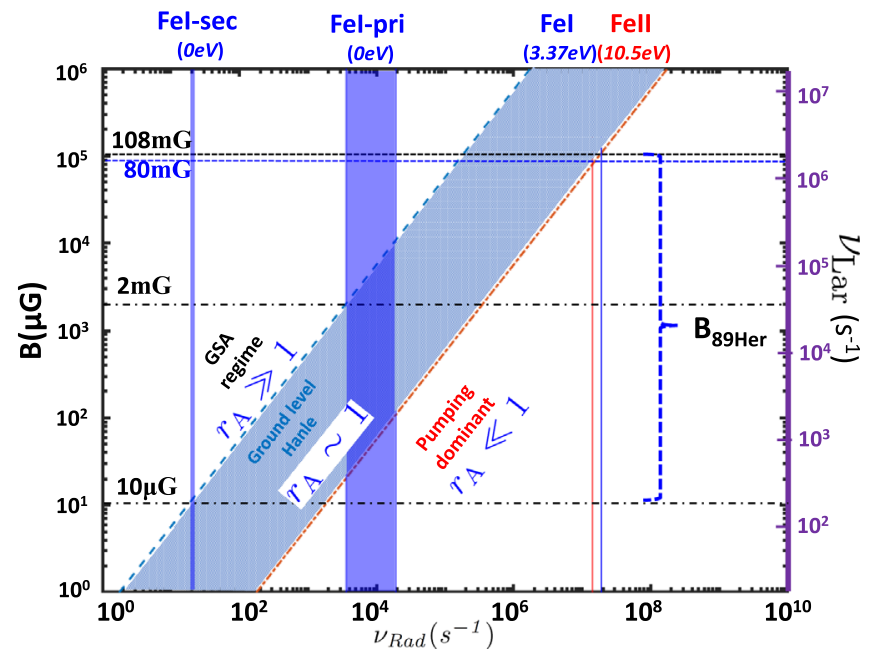

Figure 4. Magnetic field strength in $89 \mathrm{Her}$. The escaping rates from Fe I $(0 \mathrm{eV})$, and from $\mathrm{Fe} \mathrm{I}(3.37 \mathrm{eV})$ and $\mathrm{Fe} \mathrm{II}(10.5 \mathrm{eV})$, are marked at the top. The dashed-dotted lines mark the lower limits of magnetic field strength, which is estimated based on the assumption of optical pumping by the secondary and primary, respectively. The dashed lines show the upper limit of magnetic field strength derived from different transitions.

doublet (see Equation (1)). The quantity $\varepsilon_{\sigma}$ is the sign of the alignment parameter for the lower level $\sigma_{0}^{2}\left(J_{l}\right)$. The positive alignment $\varepsilon_{\sigma}=+1$ corresponds to the alignment of the angular momentum of ground states being parallel to the magnetic field, whereas the negative alignment $\varepsilon_{\sigma}=-1$ means that it is perpendicular. Therefore, the averaged magnetic field polar angle in the absorbing volume can be achieved by $\overline{\sin ^{2} \theta_{B}}=\int d V_{\text {abs }} \sigma_{0}^{2}\left(J_{l}\right) \sin ^{2} \theta_{B} / \int d V_{\text {abs }} \sigma_{0}^{2}\left(J_{l}\right)$.

In the case of the two Fe I absorption lines, the configuration parameters of these two transitions are of the same sign: $w_{J_{l} J_{u 1}}^{(2)}=\omega_{4,3}^{(2)}=0.4432 ; w_{J_{l} J_{u 2}}^{(2)}=\omega_{4,5}^{(2)}=0.2256$. Therefore, the corresponding results are

$$
\sigma_{0}^{2}\left(J_{l}\right)=\frac{-0.2176 c_{1} c_{2}+\varepsilon_{\sigma}\left(0.2256 c_{1}-0.4432 c_{2}\right)}{0.0707\left(c_{2}-c_{1}\right)},
$$

The alignment is parallel, i.e., $\varepsilon_{\sigma}=1$. $\theta_{B}$ can then be also obtained by solving the Equation (5). The resulting $\theta_{B}, \xi_{B}$ and their error bars in different phases are presented in Table 1 (see Figure 2(c) for 3D geometry). As illustrated in Figure 2(d), a dipole magnetic field on the stellar surface can support such analysis reasonably, with the average 2D magnetic field parallel to the outflow orientation on POS.

\subsection{Constraining the Magnetic Field Strength}

The magnetic field strength of 89 Her is not directly available because both the radiative alignment of the upper states and the magnetic realignment are saturated effects. Nonetheless, the magnetic field strength can be constrained by comparing the lifetime of different states (either radiative pumping rate or spontaneous emission rate) with the Larmor precession rate $\left(\omega_{\mathrm{Lar}} \sim 17.6(\mathrm{~B} / \mu \mathrm{G})\right)$. On the one hand, the radiative pumping rate from the $\mathrm{Fe}$ I ground state ${ }^{9}$ is much

\footnotetext{
9 All transitions from the given level are taken into account (both excitations and spontaneous emission) when the escaping rate from a level is calculated. The NIST Atomic Spectra Database is adopted for the Einstein coefficients to calculate the pumping rate. A total of 47 transitions from Fe I ground state and 38 transitions from the state Fe II $(10.50 \mathrm{eV})$ are included.
}

smaller than the Larmor precession rate, $\max \left\{\nu_{\mathrm{Rad}}\right\}<0.1 \nu_{\mathrm{Lar}}$. With the secondary providing the optical pumping, the magnetic field strength lower limit is $\sim 10 \mu \mathrm{G}$ (see Figure 4).

On the other hand, the synchronization between the pumping direction and the polarizations of lines from the excited states shows that the escaping rate from the excited states, which includes both radiative pumping and spontaneous emission, dominates over the Larmor precession rate in the excited states. Hence, the upper limit of the magnetic field strength can be narrowed by $10 \nu_{\mathrm{Lar}}<\max \left\{\nu_{\mathrm{Rad}}, \nu_{\mathrm{Em}}\right\}$. Utilizing the fact that Fe II $\lambda 5362.970 \AA \quad(10.50 \mathrm{eV} \rightarrow$ $12.81 \mathrm{eV}$ ) absorption line is fully radiative aligned for all of the orbital phases, we deduce that the upper limit field strength is $\sim 108 \mathrm{mG}$. Additionally, we find that the Fe I $\lambda 5586 \AA(3.37 \mathrm{eV} \rightarrow 5.59 \mathrm{eV})$ absorption line presents a more than $3 \sigma$ detectability polarization signal at the orbital phases $0.544,0.564$, and 0.988 and that the polarization direction for all those phases aligns with the radiative pumping direction rather than the magnetic field direction. This gives us an upper limit for the field strength of $\sim 80 \mathrm{mG}$. The accuracy of the field strength is increased by at least two decades compared to the previous $10 \mathrm{G}$ upper limit, constrained from the non-detection of the Zeeman effect.

\section{Discussion}

GSA is the most probable and natural explanation for the discovered polarization signals of the ground-state absorption lines that are realigned to one direction as opposed to the orbital synchronization. We provide here the mean magnetic field of the photosphere surface medium that corresponds to the central part of the absorption line. Figure 2(d) illustrates that such magnetic field is reasonable on the stellar surface. A full magnetic field tomography requires further study, which is beyond the scope of current observation precision. Higherresolution spectral observation will enable us to study the wings in the absorption line profiles, separating the emission components from outflows.

Through our analyses, we assume that the anisotropic pumping comes from the secondary (following Leone et al. 2018). However, when constraining the magnetic field strength, a narrower range might be achieved if accounting for the photons from the primary. We calculate the extended radiation field of the primary following Zhang et al. (2015) with the Lambert cosine law and limb-darkening (Claret \& Bloemen 2011) considered, and find that the lower limit for magnetic field strength can be $\sim 2 \mathrm{mG}$ (see Figure 4(a)). Additionally, we could interpret the orbital synchronization as a result of local polarized flux illuminating the medium rather than pumping from the secondary. Nonetheless, even under such an assumption, the realigned polarization direction of the two ground-state lines that we report here can still be explained by the magnetic realignment of the angular momentum on the ground state.

Moreover, beyond the optical band, GSA can be implemented with multi-frequency data ranging from ultraviolet to submillimeter to trace not only the spatial but also the temporal variations of magnetic field (Yan \& Lazarian 2007, 2012; Shangguan \& Yan 2013; Zhang \& Yan 2018). 


\section{Summary}

Polarizations of absorption lines from the ground state with a high polarization degree are discovered in the binary system of 89 Her. We conclude the following.

1. The polarization directions of the two Fe I lines show little variation across the orbital period as opposed to the polarization of many other absorption lines from the upper states in the same environment, indicating that the atoms are realigned by magnetic field in the ground state.

2. The direction of polarization in the GSA regime directly points to the $2 \mathrm{D}$ projection of the mean magnetic field in the photosphere of the primary.

3. The $90^{\circ}$ (Van Vleck) degeneracy is broken from the comparison of theoretical expectation and the observed degree of polarizations.

4. The polar angle between magnetic field and LOS is obtained from the analysis of the polarization degree of both Fe I lines.

5. The upper limit of the mean magnetic field is $\lesssim 100 \mathrm{mG}$ in the photosphere of the primary from the fact that the polarization from upper levels absorption lines are aligned by the radiation.

We acknowledge helpful communications on various aspects of this Letter with the following colleagues: F. Boulanger, S. Gao, J. Liu, R. Liu, K. Makwana, and Q. Zhu. M.G. acknowledges partial support from DESY during his visit there.

\section{Appendix A \\ Detectability of Polarization}

We have listed the detection probability for the polarization signals that we used in Table A1.

Table A1

Observing Logbook

\begin{tabular}{ccccc}
\hline \hline $\begin{array}{c}\text { HJD } \\
2400000+\end{array}$ & $\begin{array}{c}\text { Orbital } \\
\text { Phase }\end{array}$ & $\begin{array}{c}\text { Fe II 5363 } \\
\text { Det. }\end{array}$ & $\begin{array}{c}\text { Fe I 5060 } \\
\text { Det. }\end{array}$ & $\begin{array}{c}\text { Fe I 5166 } \\
\text { Det. }\end{array}$ \\
\hline 54954.125 & 0.004 & 28 & 22 & 25 \\
54955.849 & 0.010 & 24 & 17 & 18 \\
54958.121 & 0.018 & 20 & 32 & 29 \\
54959.874 & 0.021 & 17 & 13 & 13 \\
53604.753 & 0.325 & 22 & 21 & 24 \\
55109.823 & 0.544 & 29 & 19 & 21 \\
53961.777 & 0.564 & 44 & 59 & 56 \\
53775.115 & 0.916 & 20 & 15 & 34 \\
53777.096 & 0.922 & 39 & 40 & 32 \\
55229.169 & 0.958 & 36 & 25 & 27 \\
54372.821 & 0.988 & 51 & 30 & 58 \\
\hline
\end{tabular}

Note. For each line, the $\mathrm{S} / \mathrm{N}$ is determined for the polarization profiles. A polarization signal is considered true if the detection probability calculated for the null spectra inside and outside the line profile is less than 0.95 , and also if the detection probability calculated for the $\mathrm{P}$ spectra is outside of the line. All polarization signals are definitely detected as the polarization detection probability inside of the line is greater than 0.99 (Donati et al. 1997). These lines originate from the photosphere according to the analysis with the synthetic spectrum package (SYNTHE; Kurucz 2005).

\section{Appendix B \\ Equations for the GSA Reproducibility}

In this section we present the equations to describe statistical equilibrium of the evolution on upper and lower levels (see Landolfi \& Landi Degl'Innocenti 1986; Landi Degl'Innocenti \& Landolfi 2004; Yan \& Lazarian 2006):

$$
\begin{aligned}
& \dot{\rho}_{q}^{k}\left(J_{u}\right)+2 \pi i \nu_{L} g_{u} q \rho_{q}^{k}\left(J_{u}\right)=-\sum_{J_{l}} A\left(J_{u} \rightarrow J_{l}\right) \rho_{q}^{k}\left(J_{u}\right) \\
& +\sum_{J_{l} k^{\prime}}\left[J_{l}\right]\left[\delta_{k k^{\prime}} p_{k^{\prime}}\left(J_{u}, J_{l}\right) B_{l u} \bar{J}_{0}^{0}\right. \\
& \left.+\sum_{Q q^{\prime}} r_{k k^{\prime}}\left(J_{u}, J_{l}, Q, q^{\prime}\right) B_{l u} \bar{J}_{Q}^{2}\right] \rho_{-q^{\prime}}^{k^{\prime}}\left(J_{l}\right), \\
& \dot{\rho}_{q}^{k}\left(J_{l}\right)+2 \pi i \nu_{L} g_{l} q \rho_{q}^{k}\left(J_{l}\right)=\sum_{J_{u}} p_{k}\left(J_{u}, J_{l}\right)\left[J_{u}\right] A\left(J_{u} \rightarrow J_{l}\right) \rho_{q}^{k}\left(J_{u}\right) \\
& -\sum_{J_{u} k^{\prime}}\left[\delta_{k k^{\prime}} B_{l u} \bar{J}_{0}^{0}+\sum_{Q q^{\prime}} s_{k k^{\prime}}\left(J_{u}, J_{l}, Q, q^{\prime}\right) B_{l u} \bar{J}_{Q}^{2}\right] \rho_{-q^{\prime}}^{k^{\prime}}\left(J_{l}\right),(\mathrm{B} 2)
\end{aligned}
$$

in which

$$
\begin{aligned}
& p_{k}\left(J_{u}, J_{l}\right)=(-1)^{J_{u}+J_{l}+1}\left\{\begin{array}{ccc}
J_{l} & J_{l} & k \\
J_{u} & J_{u} & 1
\end{array}\right\}, \\
& p_{0}\left(J_{u}, J_{l}\right)=\frac{1}{\sqrt{\left[J_{u}, J_{l}\right]}}, \\
& r_{k k^{\prime}}\left(J_{u}, J_{l}, Q, q\right)=\left(3\left[k, k^{\prime}, 2\right]\right)^{\frac{1}{2}} \\
& \times\left\{\begin{array}{ccc}
1 & J_{u} & J_{l} \\
1 & J_{u} & J_{l} \\
2 & k & k^{\prime}
\end{array}\right\}\left(\begin{array}{ccc}
k & k^{\prime} & K \\
q & q^{\prime} & Q
\end{array}\right), \\
& s_{k k^{\prime}}\left(J_{u}, J_{l}, Q, q\right)=(-1)^{J_{l}-J_{u}+1}\left[J_{l}\right]\left(3\left[k, k^{\prime}, K\right]\right)^{\frac{1}{2}} \\
& \times\left(\begin{array}{ccc}
k & k^{\prime} & 2 \\
q & q^{\prime} & Q
\end{array}\right)\left\{\begin{array}{ccc}
1 & 1 & 2 \\
J_{l} & J_{l} & J_{u}
\end{array}\right\}\left\{\begin{array}{ccc}
k & k^{\prime} & 2 \\
J_{l} & J_{l} & J_{l}
\end{array}\right\} .
\end{aligned}
$$

The quantities $J_{u}$ and $J_{l}$ are the total angular momentum quantum numbers for the upper and lower levels, respectively. The quantities $\rho_{q}^{k}$ and $\bar{J}_{Q}^{K}$ are irreducible density matrices for the atoms and the incident radiation, respectively. The $6-j$ and $9-j$ symbols are represented by the matrices with " \{\} ," whereas $3-j$ symbols are indicated by the matrices with "()." The second terms on the left side of Equations (B1) and (B2) stand for the magnetic realignment. The two terms on the right side represent spontaneous emissions and the excitations from lower levels. Note that the symmetric processes of spontaneous emission and magnetic realignment conserve $k$ and $q$. Therefore, the steady state occupations of atoms on the ground state are obtained by setting the left side of Equations (B1) and B2 
zero:

$$
\begin{aligned}
& 2 \pi i \rho_{q}^{k}\left(J_{l}\right) q g_{l} \nu_{L}-\sum_{J_{u} k^{\prime}}\left\{p_{k}\left(J_{u}, J_{l}\right) \frac{\left[J_{u}\right]}{\sum_{J^{\prime \prime} l} A^{\prime \prime} / A+i \Gamma^{\prime} q}\right. \\
& \quad \times \sum_{J_{l}^{\prime}} B_{l u}\left[J_{l}^{\prime}\right]\left[\delta_{k k^{\prime}} p_{k^{\prime}}\left(J_{u}, J_{l}^{\prime}\right) \bar{J}_{0}^{0}+\sum_{Q q^{\prime}} r_{k k^{\prime}}\left(J_{u}, J_{l}^{\prime}, Q, q^{\prime}\right) \bar{J}_{Q}^{2}\right] \\
& \left.\quad-\delta_{J_{l} J_{l}^{\prime}}\left[\delta_{k k^{\prime}} B_{l u} \bar{J}_{0}^{0}+\sum_{Q q^{\prime}} B_{l u} s_{k k^{\prime}}\left(J_{u}, J_{l}, Q, q^{\prime}\right) \bar{J}_{Q}^{2}\right]\right\} \rho_{-q^{\prime}}^{k^{\prime}}\left(J_{l}^{\prime}\right) \\
& \quad=0
\end{aligned}
$$

where $\Gamma^{\prime}$ equals $2 \pi \nu_{L} g_{u} / A$. Magnetic realignment on the levels in excited states can be neglected because the escape from the excited states is much higher than magnetic precession rate in this environment. As a result, $\Gamma^{\prime} \simeq 0$. On the other hand, the magnetic precession rate is much higher than the photon excitation rate of the atoms on the ground state in the diffuse media of the interstellar and intergalactic medium $\left(\nu_{L} \gg B_{l u} \bar{J}_{0}^{0}\right)$. Thus, we applied Equation (B4) to the five magnetically aligned sublevels on the ground state of Fe I $\left(3 d^{6} 4 s^{2}{ }_{a}^{5} D: 0 \mathrm{eV}(J=4)\right.$, $0.05 \mathrm{eV}(J=3), 0.09 \mathrm{eV}(J=2), 0.11 \mathrm{eV}(J=1)$, and $0.12 \mathrm{eV}$ $(J=0))$ by setting $q=0$ so that the first term on the left equals 0 . By solving the above equations, the alignment parameter $\left(\sigma_{0}^{2}\left(J_{l}\right)\right)$ under the influence of GSA is obtained.

\section{ORCID iDs}

Heshou Zhang (ib https://orcid.org/0000-0003-2840-6152 Manuele Gangi (iD https://orcid.org/0000-0002-8364-7795 Francesco Leone (iD https://orcid.org/0000-0001-7626-3788 Huirong Yan (D) https://orcid.org/0000-0003-2560-8066

\section{References}

Akras, S., Ramírez Vélez, J. C., Nanouris, N., et al. 2017, MNRAS, 466, 2948 Brossel, J., Kastler, A., \& Winter, J. 1952, J. Phys. Radium., 13, 668 Bujarrabal, V., van Winckel, H., Neri, R., et al. 2007, A\&A, 468, L45 Claret, A., \& Bloemen, S. 2011, A\&A, 529, A75

Cohen-Tannoudji, C., Dupont-Roc, J., Haroche, S., \& Laloë, F. 1969, PhRvL, 22, 758

Donati, J.-F., Catala, C., Landstreet, J. D., \& Petit, P. 2006, in ASP Conf. Ser. 358, Solar Polarization 4, ed. R. Casini \& B. W. Lites (San Francisco, CA: ASP), 362

Donati, J.-F., Semel, M., Carter, B. D., Rees, D. E., \& Collier Cameron, A. 1997, MNRAS, 291, 658

Gangi, M., \& Leone, F. 2019, AN, 340, 409

Happer, W. 1972, RvMP, 44, 169

Hawkins, W. B. 1955, PhRv, 98, 478

Hawkins, W. B., \& Dicke, R. H. 1953, PhRv, 91, 1008

House, L. L. 1974, PASP, 86, 490

Kastler, A. 1950, J. Phys. Radium, 11, 255

Kipper, T. 2011, BaltA, 20, 65

Kurucz, R. L. 2005, MSAIS, 8, 14

Landi Degl'Innocenti, E. 1984, SoPh, 91, 1

Landi Degl'Innocenti, E., \& Landolfi, M. 2004, Polarization in Spectral Lines, Astrophysics and Space Library, Vol. 307 (Dordrecht: Kluwer)

Landolfi, M., \& Landi Degl'Innocenti, E. 1986, A\&A, 167, 200

Leone, F., Avila, G., Bellassai, G., et al. 2016, AJ, 151, 116

Leone, F., Cecconi, M., Cosentino, R., et al. 2014, Proc. SPIE, 9147, 91472F

Leone, F., Gangi, M., Giarrusso, M., et al. 2018, MNRAS, 480, 1656

Piskunov, N., Snik, F., Dolgopolov, A., et al. 2011, Msngr, 143, 7

Sabin, L., Wade, G. A., \& Lèbre, A. 2015, MNRAS, 446, 1988

Shangguan, J., \& Yan, H. 2013, Ap\&SS, 343, 335

Strassmeier, K. G., Woche, M., Ilyin, I., et al. 2008, Proc. SPIE, 7014, 70140N

Van Vleck, J. H. 1925, PNAS, 11, 612

Varshalovich, D. A. 1971, SvPhU, 13, 429

Waters, L. B. F. M., Waelkens, C., Mayor, M., \& Trams, N. R. 1993, A\&A, 269, 242

Yan, H., \& Lazarian, A. 2006, ApJ, 653, 1292

Yan, H., \& Lazarian, A. 2007, ApJ, 657, 618

Yan, H., \& Lazarian, A. 2008, ApJ, 677, 1401

Yan, H., \& Lazarian, A. 2012, JQSRT, 113, 1409

Zhang, H., \& Yan, H. 2018, MNRAS, 475, 2415

Zhang, H., Yan, H., \& Dong, L. 2015, ApJ, 804, 142 